Journal of Engineering and Applied Sciences 14 (11): 3625-3629, 2019

ISSN: 1816-949X

(C) Medwell Journals, 2019

\title{
MRI Technique Based Detection and Classification of Brain Tumor using Support Vector Machine (SVM) and k-Nearest Neighbor (kNN)
}

\author{
Hassan Jassim Motlak \\ Department of Electrical Engineering, College of Engineering, University of Babylon, Babylon, Iraq
}

\begin{abstract}
This study presents a system has the ability to detect and classify brain cancer effectively and efficiently based-on processing images that are combined with a Magnetic Resonance Imaging (MRI) technique. MRI has high features in dealing with human life such as safety, reliability and it is ability to image in any plane. The proposed system starts with the preprocessing of images includes resizing and enhancement of gray images of brain tumor. Textures features of the brain tumor are extracted using two algorithms called GCLM and k-means. The final stage to classify the tumor if benign or malign was accomplished using two techniques are k-Nearest Neighbor algorithm (kNN) and Support Vector Machine (SVM). The simulation results using MATLAB environment, showed that the accuracy of SVM classifier was better than KNN in the classification of brain tumor where the results are 79 and $73 \%$, respectively. But the value of specificity of the system for kNN method was higher than SVM and the results are 87.5 and $61 \%$, respectively.
\end{abstract}

Key words: MRI technique, brain tumor, support vector machine, k-nearest neighbor, accuracy, MATLAB

\section{INTRODUCTION}

Medical image processing specially, MRI imaging modalities is the most difficult and creative field. X-ray is a 3D non-obtrusive imaging methodology which is most appropriate for delicate tissue variation from the norm identification. The MRI imaging system is the best for identification of cerebrum tumors because of its high determination and capacity to indicate clear mind structures, tumor's size and area (Wang et al., 2016). From MRI mind picture, the valuable data is gotten for the recognition of tumors. These days there are a few approachs for arranging MRI pictures, for example, viz. fluffy techniques, Bolster Vector Machine (SVM), locale developing strategy, neural systems information based strategies, watershed division strategies and different other division strategies are accounted by Mahmoud and Obaidellah (2017), Suhag and Saini (2015), Kaushik et al. (2014), Nichat and Ladhake (2016), Kumar and Mahavir (2015), Nithyapriya and Sasikumar (2014), Chandrakala and Sumathi (2014), Madheswaran and Dhas (2015), Moumita and Ghosh (2013), Motlak and Hakeem (2017), Pan et al., 2010).

In this study, brain tumor are detected and classified using two classification techniques. The first technique based on SVM methodology and second technique based-on kNN algorithm. Simulation results showed that, an efficient and sensitive system in detection of brain tumor using MRI gray images. Comparison between results of kNN and SVM classifiers, proved that the SVM is more accurate and sensitive than $\mathrm{kNN}$.

\section{MATERIALS AND METHODS}

Proposed system methodology: This study clarifies the proposed framework outline and strategy. The proposed strategy comprises of number of stages which are preprocessing, highlight extraction from MRI informational index utilizing GLCM and k-mean. For grouping reason, two classifier are utilized, the principal compose is SVM is utilized for arrangement of cerebrum pictures as ordinary or anomalous. The second kind of the classifier is kNN. Figure 1 demonstrates the stream graph for the proposed technique.

Classification using SVM: So as to group the information picture as typical or unusual, we connected SVM. SVM is a methodical strategy for two class issues. The SVM classifier is utilized as a part of many research regions, since, it gives superior in design acknowledgment and picture handling errands. SVM is in all likelihood utilized as a part of issues with little preparing dataset and high dimensional component space. Like neural systems, SVM needs two phases, preparing and testing. The SVM can be prepared by highlights given as a contribution to its learning calculation. Amid preparing, the SVM finds the reasonable edges between two classes. Highlights are named by class affiliated with particular class (Gunn, 1998).

ANN has numerous disadvantages, for example, having nearby minima and the choice of number of 


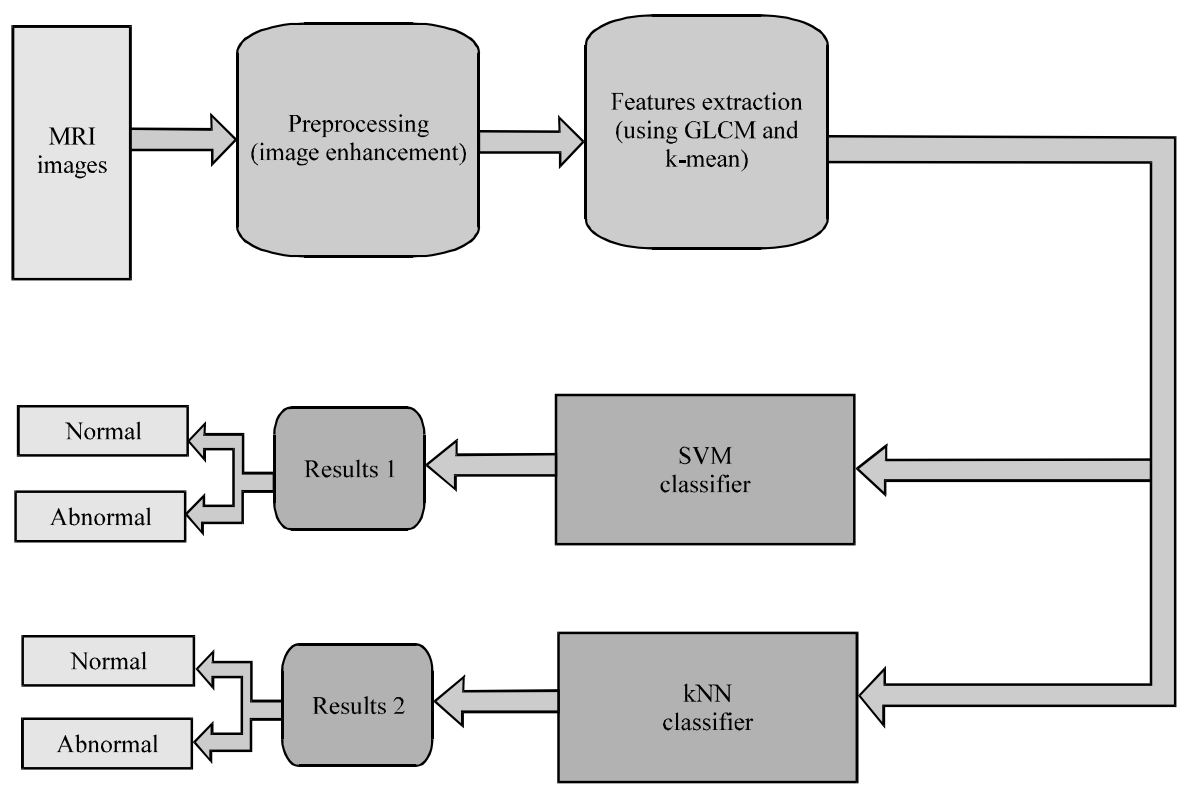

Fig. 1: The flow diagram of the proposed system

neurons for every issue. SVM possesses no nearby minima and by starting the possibility of hyper planes, it defeats the issue of neurons choice.

In our SVM input information is mapped into higher dimensional space utilizing RBF bit. In this changed space, a hyper plane straight classifier is connected using those examples vectors that are nearest to the choice limit. Let $\mathrm{m}$-dimensional sources of info $\mathrm{x}_{\mathrm{i}}(\mathrm{I}=1, \ldots, \mathrm{M})$ have a place with class 1 or 2 and the related marks be $y_{i}=1$ for class 1 and -1 for class 2 . Choice capacity for SVM is:

$$
D(x)=w^{t} x+b
$$

Where:

$\mathrm{w}=$ An m-dimensional vector

$\mathrm{b}=$ A scalar

The separating hyper plane satisfies:

$$
\mathrm{y}_{\mathrm{i}}\left(\mathrm{w}^{\mathrm{t}} \mathrm{x}_{\mathrm{i}}+\mathrm{b}\right) \geq 1 \text { for } \mathrm{i}=1, \ldots, \mathrm{M}
$$

The separation between the isolating hyper plane D (x) $=0$ and the preparation datum closest to the hyper plane is known as the edge. The hyper plane $D(x)=0$ that has the greatest edge is known as the ideal isolating hyper plane (Alfonse and Salem, 2016).

This sharp imperative on the parameterization is desirable over options in disentangling the detailing of the issue. In words it expresses that: the standard of the weight vector ought to be equivalent to the opposite of the separation, of the closest point in the informational

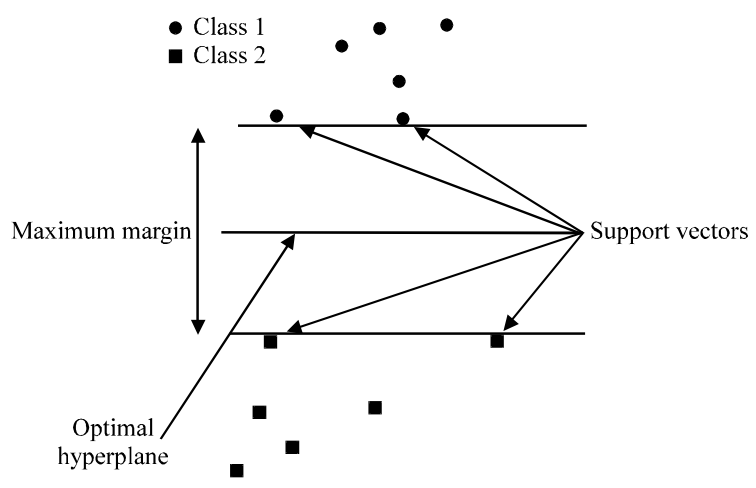

Fig. 2: Linear SVM

index to the hyper plane. The thought is delineated in Fig. 2 where the separation from the closest point to each hyper plane is demonstrated (Ghotekar and Mahajan, 2016).

MRI technique:Attractive Reverberation Imaging (MRI) is a spectroscopic imaging method utilized as a part of restorative settings to create pictures of within the human body. X-ray depends on the standards of atomic attractive reverberation (NMR) which is a spectroscopic method used to acquire tiny compound and physical information about particles. In 1977 the primary MRI exam was performed on an individual. It took $5 \mathrm{~h}$ to deliver one picture. The attractive reverberation imaging is refined through the ingestion and outflow of vitality of the radio Recurrence (RF) scope of the electromagnetic range. Uses non ionizing radiation, dissimilar to $\mathrm{X}$-beams. Capacity to 
(a)

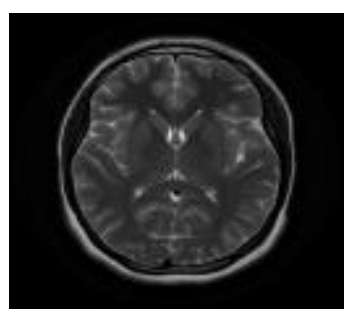

(b)

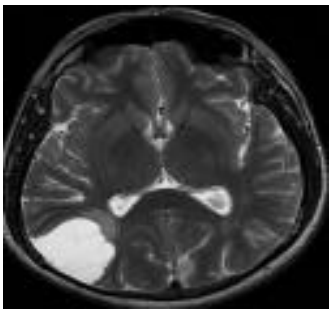

Fig. 3: a, b) Normal (on the left) and tumor (on the right) MRI image

picture in any plane, not at all like CT filters. Low occurrences of reactions. Capacity to analyze, imagine and assess different sicknesses. The main better approach to see the internal parts of your body is to $\mathrm{Cu}$ you open. A magnet which delivers a ground-breaking uniform attractive field. Inclination magnets which are much lower in quality. Gear to transmit radio recurrence (RF). A great PC framework which deciphers the signs transmitted by the curls. The information of ordinary and strange mind tumor were gathered are appeared in Fig. 3 (NIST., 2018).

\section{RESULTS AND DISCUSSION}

Simulation results: Table 1 shows detection results of the proposed system. The texture features are extracted based on different algorithms. The final decision of detection process are normal and abnormal samples. It can be seen that the detection rate of these samples using SVM with GLCM algorithm is better than $\mathrm{kNN}$ with GLCM or Gabor filter. Table 2 shows the specificity, sensitivity and accuracy results obtained using SVM and $\mathrm{kNN}$ classifiers. Results showed that, the proposed system is more accurate and sensitive in case SVM classifier but it is more specificity in case $\mathrm{kNN}$ classifier. Moreover, the system accuracy is increased with the increasing of the features extraction.

Figure 4 illustrate the SVM classifier performance based on hyper plane using two approaches. The first approach is images resizing and second approach using segmented image. It can be seen that the separate data in in second approach is better than first one where the training data is linearly separable and maximize the distance between normal and abnormal data. This linear classifier is termed the optima separating hyper plane. Intuitively, we would expect this boundary to generalize well as opposed to the other possible boundaries. Figure 5 shows simple comparison between SVM and $\mathrm{kNN}$ classifier at 2 feature extraction methods 1 represent texture features at resized image 2 represent texture
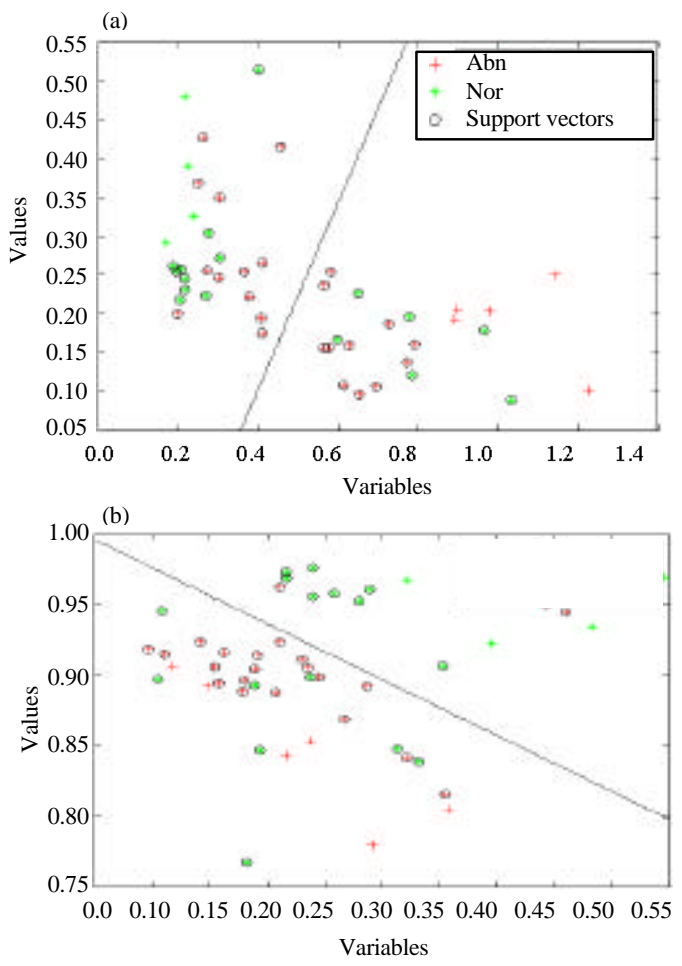

Fig. 4: Support vectors performance based on two features: a) Texture features using resized image and b) Texture features using segmented image

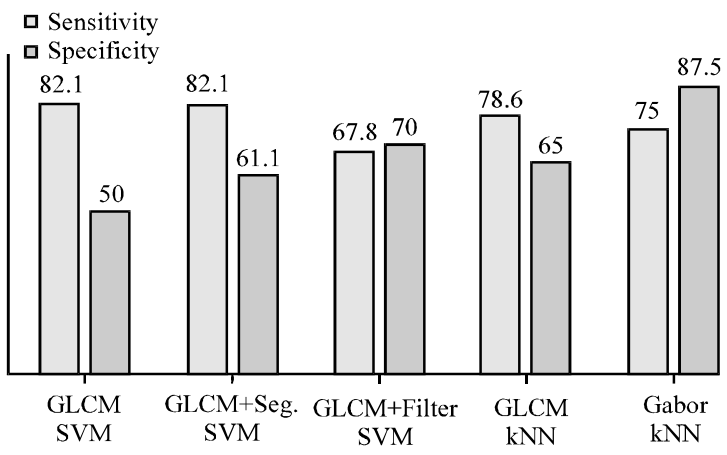

Fig. 5: Chart present comparison of sensitivity and specificity values for SVM and $\mathrm{kNN}$ classifiers

features at segmented image are obtained. Where texture features showed best sensitivity using SVM classifier is $82.1 \%$ and better spasticity is achieved by $\mathrm{kNN}$ was $87.5 \%$.

The comparison between results of the proposed system using SVM and kNN classifiers with previous Ghotekar and Mahajan (2016) are summarized in Table 3. We can note that, the specificity of the proposed system is better than by Gunn (1998). But the other 
Table 1: Detection results of proposed system

\begin{tabular}{llccc}
\hline Classifier & Features & Training samples & Abnormal correctly detected & Normal correctly detected \\
SVM & GlCM (16) & 43 & 23 & 9 \\
SVM & GlCM (16) & 43 & 23 & 11 \\
kNN & GlCM (16) & 48 & 19 & 14 \\
kNN & GlCM (16) & 48 & 22 & 13 \\
kNN & Gabor (40) & 48 & 21 & 14 \\
\hline
\end{tabular}

Table 2: Classification results of the proposed system

\begin{tabular}{llcclll}
\hline Classifier & Feature algorithm & Number of features & Sensitivity & Specificity & Accuracy & k-fold \\
\hline SVM & Glcm & 16 & 82.1 & 50 & 75 & 10 \\
SVM & Glcm k-mean & 16 & 82.1 & 61.1 & 79 & 5 \\
kNN & Glcm+Median & 16 & 67.8 & 70 & 68.75 & - \\
kNN & Glcm+Image resizing & 16 & 78.6 & 65 & 73 & - \\
kNN & Gabor filter & 40 & 75.0 & 87.5 & 73 & - \\
\hline
\end{tabular}

Table 3: Summarized comparison results of the proposed system using SVM and kNN with previous work by Ghotekar and Mahajan (2016)

\begin{tabular}{|c|c|c|c|}
\hline Parameters & $\begin{array}{l}\text { The proposed system } \\
\text { using SVM+k-mean }\end{array}$ & $\begin{array}{l}\text { The proposed system } \\
\text { using } k N N+\text { Gabor }\end{array}$ & $\begin{array}{l}\text { The work } \\
\text { Ghotekar and } \\
\text { Mahajan (2016) }\end{array}$ \\
\hline Accuracy & 79 & 73.0 & 83.30 \\
\hline Sensitivity & 82.1 & 78.6 & 91.52 \\
\hline Specificity & 70 & 87.5 & 67.74 \\
\hline
\end{tabular}

parameters such as accuracy and sensitivity is approximate with that by Ghotekar and Mahajan (2016).

\section{CONCLUSION}

Support vector machines are an appealing way to deal with information displaying. They join speculation control with a strategy to address the scourge of dimensionality. The plan brings about a worldwide quadratic enhancement issue with box requirements which is promptly explained by inside point techniques. In order issues speculation control is acquired by boosting the edge which compares to minimization of the weight vector in an accepted structure. The arrangement is gotten as an arrangement of help vectors that can be scanty. These lie on the limit and accordingly condense the data required to isolate the information. The minimization of the weight vector can be utilized as a basis in relapse issues with a changed misfortune work. Different calculations are utilized for separating surfaces highlights of the cerebrum tumor from MRI pictures are tended to. Two classifiers SVM and $\mathrm{kNN}$ systems are utilized for distinguishing and characterizing ordinariness and variation from the norm of cerebrum tumor. Reenactment comes about delineated that, productive framework as indicated by aftereffects of exactness, affectability and specificity. Examination between two classifier demonstrated that that the SVM classifier is more exact than $\mathrm{kNN}$ classifier. In spite of the fact that the $\mathrm{kNN}$ is better in specificity.

\section{ACKNOWLEDGEMENT}

This research was supported by the Ministry of Higher Education and Scientific Research and University of Babylon, Iraq.

\section{REFERENCES}

Alfonse, M. and A.B.M. Salem, 2016. An automatic classification of brain tumors through MRI using support vector machine. Egypt. Comput. Sci. J., 40: 11-21.

Chandrakala, D. and S. Sumathi, 2014. Image classification based on color and texture features using FRBFN network with artificial bee colony optimization algorithm. Intl. J. Comput. Appl., 98: 19-29.

Ghotekar, B. and K.J. Mahajan, 2016. Brain tumor detection and classification using SVM. National Conf. Innovative Trends Sci. Eng., 4: 180-182.

Gunn, S.R., 1998. Support vector machines for classification and regression. MSc Thesis, School of Electronics and Computer Science, University of Southampton, Southampton, England.

Kaushik, D., U. Singh, P. Singhal and V. Singh, 2014. Brain tumor segmentation using Genetic algorithm. Intl. J. Comput. Appl., 5: 13-15.

Kumar, A. and B. Mahavir, 2015. A novel approach for brain tumor detection using support vector machine k-means and pca algorithm. Intl. J. Comput. Sci. Mob. Comput., 4: 457-474.

Madheswaran, M. and D.A.S. Dhas, 2015. Classification of brain MRI images using support vector machine with various Kernels. Biomed. Res., 26: 505-513. 
Mahmoud, A. and U.B. Obaidellah, 2017. Artificial intelligence techniques for cancer detection and classification: Review study. Eur. Sci. J., 13: 342-370.

Motlak, H.J. and S.I. Hakeem, 2017. Detection and classification of breast cancer based-on terahertz imaging technique using artificial neural network and k-nearest neighbor algorithm. Intl. J. Appl. Eng. Res., 12: 10661-10668.

Moulick, H.N. and M. Ghosh, 2013. Medical image processing using a simd array processor and neural networks. Intl. J. Eng. Inventions, 2: 56-64.

NIST., 2018. Neuro imaging and surgical technologies lab. National Institute of Standards and Technology, Gaithersburg, Maryland, USA. http://nist. mni.mcgill.ca/

Nichat, A.M. and S.A. Ladhake, 2016. Brain tumor segmentation and classification using modified FCM and SVM classifier. Intl. J. Adv. Res. Comput. Commun. Eng., 5: 73-76.
Nithyapriya, G. and C. Sasikumar, 2014. Detection and segmentation of brain tumors using AdaBoost SVM. Intl. J. Innovative Res. Comput. Commun. Eng., 2: 2323-2328.

Pan, R., S. Zhao and J. Shen, 2010. Terahertz spectra applications in identification of illicit drugs using support vector machines. Procedia Eng., 7: 15-21.

Suhag, S. and L.M. Saini, 2015. Automatic brain tumor detection and classification using SVM classifier. Proceedings of the ISER 2nd International Conference on ISER, July 19, 2015, Singapore, ISBN:978-93-85465-51-2, pp: 55-59.

Wang, S., Y. Zhang, T. Zhan, P. Phillips and Y.D. Zhang et al., 2016. Pathological brain detection by artificial intelligence in magnetic resonance imaging scanning (invited review). Prog. Electromagnet. Res., 156: 105-133. 